\title{
Cloning and expression of cold-inducible RNA binding protein in domestic yak (Bos grunniens)
}

\author{
K. Yang*, Q. Zhang*, Z. Wen, Y. Pan, S. Yu, J. He, X. Yang, P. Liu, Y. Cui \\ Laboratory of Animal Anatomy and Tissue Embryology, Department of Basic Veterinary Medicine, Faculty of Veterinary \\ Medicine, Gansu Agricultural University, Lanzhou, Gansu, China
}

[Received 15 September 2015; Accepted: 10 November 2016]

\begin{abstract}
Cold-inducible RNA binding protein (CIRP) is over-expressed during cold and many other stresses, and could regulate the adaptation to hypothermia. In the present investigation, the objective was to determine the expression of CIRP in adult yak heart, liver, spleen, lung, kidney, brain, ovary, testis and skin by relative quantitative real time polymerase chain reaction (RT-PCR), Western blot and immunohistochemistry from MRNA and protein levels. The CIRP open reading frame encoding was cloned from the domestic yak brain. Results of RT-PCR and Western blot showed the high expression level of CIRP in the heart, kidney, brain, testis and skin, and the lower expression level of CIRP in the lung. Immunohistochemical staining showed CIRP was expressed in the nucleus of neuronal cells, spermatogonia, primary spermatocytes and epidermal cells, and in the cytoplasm of the residual tissues. These observations may provide new data to understand and further study the important role of CIRP protein in the plateau adaptation of the domestic yak on long-term evolution. (Folia Morphol 2016; 75, 4: 460-466)
\end{abstract}

Key words: yak, cold-inducible RNA binding protein, expression, adaptation, RT-PCR, Western blot, immunohistochemistry

\section{INTRODUCTION}

The Qinghai-Tibetan plateau is regarded as "the roof of the world" and bound by the Kunlun and Aljin mountains in the northeast and the Himalayas in the Southwest. It towers over Southwestern China covering more than 2.5 million $\mathrm{km}^{2}$ at an altitude of $4000 \mathrm{~m}$ above sea level. Yaks are found extensively on the Qinghai-Tibetan plateau at altitudes from 2000 to $5000 \mathrm{~m}$ with a cold, semi-humid climate and hypoxia environment. Additionally, the total yak population is estimated about 14.2 million, of which 13.3 million are in China [14].

Generally, hypothermia, hypoxia and strong UV radiation of plateau are the vital factors determining the distribution, stocking density and the growth rate of animals. However, the native animal and people after a long evolution have a good adaptation for the plateau environment and form their own adaptation mechanisms which have been reserved as the biological characteristics. The yak is a typical representative of native plateau animals. Anatomically, adaptation in low oxygen content about $40 \%$ oxygen of the air results from a large lung, large chest (14-15 pairs of thoracic ribs) and a large heart relative to their overall body size [4]. Thick and long hair is suitable for the environment of strong ultraviolet ray and cold weather $[12,14]$.

Moreover, for animals the change in $\mathrm{O}_{2}$ concentration and temperature are the fundamental physiologic stimuluses leading to both acute and chronic responses. Intracellular temperature and $\mathrm{O}_{2}$ concentrations are maintained within a narrow range due to the risk of metabolic demise from hypothermia and hypoxia or of oxidative damage from hyperther-

Address for correspondence: Dr Yan Cui, Laboratory of Animal Anatomy and Tissue Embryology, Department of Basic Veterinary Medicine, Faculty of Veterinary Medicine, Gansu Agricultural University, Gansu, Lanzhou 730070, RP China, tel: +8613609310891, e-mail: cuiyan369@sina.com *These authors contributed equally to this article. 
mia and hyperoxia. However acute responses often undertake changes in the activity of preexisting proteins, chronic responses invariably involve changes in gene expression. Cold-inducible RNA binding protein (CIRP) is activated by hypothermia, hypoxia and UV radiation; moreover, several reports have noted that CIRP activation could also be found in response to stresses related with tumour [9]. The CIRP protein is composed of 172 amino acids, which contains two clearly defined domains. The first is an RNA binding domain (RMM motif), which is well conserved across different species and involved in RNA binding. The second is a glycine-rich domain (RGG motif) with an undefined function [5]. Except for its role in the cell growth inhibition caused by cold, CIRP as a critical factor has been involved in the tumour suppressor, pathogenesis of tumour genesis, and played a regulatory role in stresses such as ultraviolet radiation and tissue hypoxia [5]. Also there are some studies on the CIRP in the human [2], amphibian [11], rat and mouse $[8,15,16]$. Despite all this, there is no research about the CIRP of native plateau species which live under extreme hypoxia, hypothermia and UV radiation conditions. The objective of our study was to characterise the CIRP CDNA of the yak and to detect its mRNA and protein expression in various tissues (heart, liver, spleen, lung, kidney, brain, ovary, testis and skin) for further functional and ecological adaptation studies.

\section{MATERIALS AND METHODS}

\section{Collection of different tissues}

Tissues including the heart, liver, spleen, lung, kidney, brain, ovary, testis and skin were taked in 5 male and 5 female adult (3-4 years) yaks in September, 2014. The protocol used for animal use and euthanasia were approved by the State Forestry Administration and complied with the Guide for the Care and Use of Laboratory Animals by the Ministry of Science and Technology of the People's Republic of China. For mRNA and protein analysis, fresh tissues were collected $10 \mathrm{~min}$ after the yak slaughter. They were washed with DEPC treated water $(0.1 \%)$ and stored in liquid nitrogen. For immunohistochemistry, small pieces of tissues were fixed with $4 \%$ paraformaldehyde phosphate buffer ( $\mathrm{pH}$ 7.3).

\section{RNA isolation and gene cloning}

Total RNA was extracted from different tissues using TRIzol reagent (Omega, Norcross, GA, USA) ac- cording to the manufacturer's instructions. RNA was eluted in $30 \mu \mathrm{L}$ RNase-free water and its quality and quantity were assessed with an Experion Automated Electrophoresis Station (BioRad, Munich, Germany) using Experion RNA StdSens Analysis Kit (BioRad, Munich, Germany). The RNA quality indicator (RQI) ranging between 5 and 10 was used [3]. RNA was reverse transcribed with $100 \mu \mathrm{M}$ Oligo-dT18 primers using the RevertAid first Strand cDNA Synthesis Kit (Promega, Mannheim, Germany) according to the manufacturer's protocol.

The domestic yak CIRP primers were designed according to the CIRP CDNA sequence of Bos taurus (Gen-Bank Accession No. NM_001034278.1), Macaca fascicularis (GenBank Accession No. AB170797.1), Sus scrofa (GenBank Accession No. HQ908795.1), Macaca mulatta (GenBank Accession No. NM_001261316.1), Homo sapiens (GenBank Accession No. D78134.1), and Pan troglodytes (GenBank Accession No. AK306150.1) (Fig. 1). The gene were cloned into pGEM T-Easy Vector (Promega, Mannheim, Germany).

\section{mRNA expression analysis with RT-PCR}

To relative quantitative gene expression, the real time polymerase chain reaction (RT-PCR) primers for yak CIRP were designed based on the sequences of $B O S$ grunniens (Gen-Bank Accession No. KF682140) and the RT-PCR primers for $\beta$-actin were designed based on the sequences of Bos grunniens (GenBank Accession No. DQ838049.1). The information about RT-PCR primers for yak CIRP and internal control $\beta$-actin is listed in Figure 1. $200 \mathrm{ng}$ of total CDNA, in a $20-\mu \mathrm{L}$ reaction volume was analysed in LightCycler480 thermocycler (Roche, Germany) using SYBR Premix DimerEraser (Promega, Mannheim, Germany) and $100 \mathrm{nM}$ of reverse and forward primer each [10]. All RT-PCRs were run in duplicates with a non-template control to check for the contaminations. The conditions of RT-PCR were $3 \mathrm{~min}$ at $95^{\circ} \mathrm{C}$ and 40 cycles of each $95^{\circ} \mathrm{C}$ for $20 \mathrm{~s}$, $60^{\circ} \mathrm{C}$ for $20 \mathrm{~s}$ and $72^{\circ} \mathrm{C}$ for $15 \mathrm{~s}$. Melting curve analysis was performed from $65^{\circ} \mathrm{C}$ to $95^{\circ} \mathrm{C}$ in $0.5^{\circ} \mathrm{C}$ steps each lasting $5 \mathrm{~s}$ to confirm the presence of a single product and absence of primer-dimers.

\section{The protein extraction and analysis with Western blot}

Briefly, the tissues were washed 3 times in ice-cold phosphate-buffered saline (PBS), and the total proteins were extracted with in extraction buffer (Beyotime, China). It was separated on $10 \%$ SDS-PAGE gel 


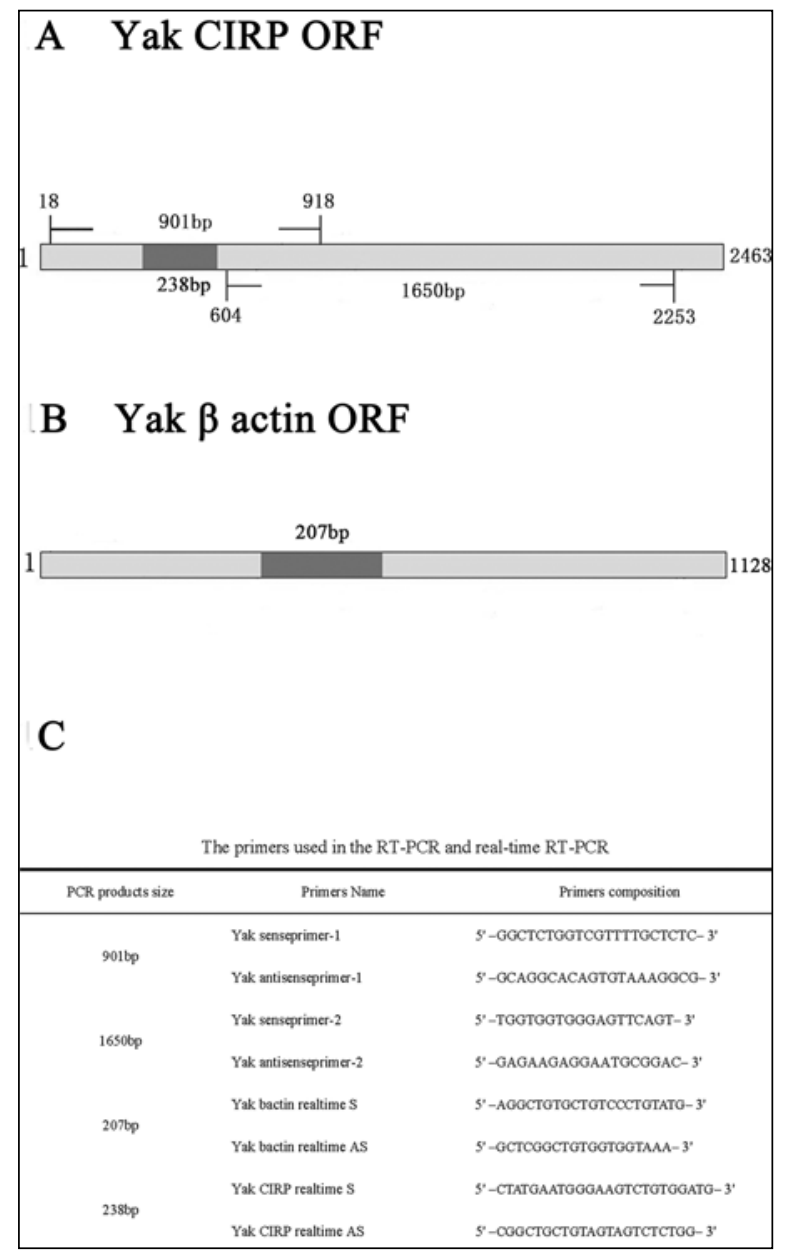

Figure 1. Polymerase chain reaction (PCR) primers used in the real time (RT)-PCR and real-time RT-PCR. A. The domestic yak coldinducible RNA binding protein (CIRP) open reading frame (ORF); B. The domestic yak $\beta$-actin ORF. The blue part is the real-time RT-PCR products of the whole ORFs; C. The primers used in the RT-PCR and real-time RT-PCR.

using a Bio-Rad apparatus and then transferred electrophoretically onto enhanced chemiluminescence (ECL) polyvinylidene fluoride (PVDF) membranes (Amersham, USA) using a Bio-Rad Mini Trans-Blot Cell (Bio-Rad, Hercules, CA, USA). The membranes were then blocked with $5 \%$ nonfat dry milk and $0.1 \%$ Tween 20 in Tris-buffered saline and subsequently incubated with Anti-CIRP (ab106230, Abcam; dilution 1:1000 in PBS) in Tris-buffered saline at $37^{\circ} \mathrm{C}$ for $2 \mathrm{~h}$, which contained $5 \%$ non-fat dry milk and $0.1 \%$ Tween 20 . Then, the membrane was washed 3 times in Tris-buffered saline, each time for $10 \mathrm{~min}$. The horseradish peroxidase conjugated mouse antigoat IgG (sc-2354, Santa Cruz; dilution 1:8000 in PBS containing $1 \%$ bovine serum albumin (BSA) was used to detect conjugates and the antibody-antigen complexes. The membrane was washed 3 times in Tris-buffered saline, each time for $10 \mathrm{~min}$, followed by use of an ECL detection kit (Beyotime, China). Intensity of the bands on the blots was measured by a densitometric analysis system (Bio-Rad). Intensity of $\beta$-actin bands were used for normalisation.

\section{Immunohistochemistry and detection}

The tissues of yaks were dissected and fixed in $4 \%$ paraformaldehyde phosphate buffer $(\mathrm{pH} 7.3)$ for 2 weeks, followed by embedding in paraffin. Sections of $5 \mu \mathrm{m}$ thickness were prepared and placed on microscope slides (Superfrost Plus, Thermo Scientific, Braunschweig). Sections were deparaffinised in $x y-$ lene and rehydrated in decreasing concentrations of ethanol before rinsing in PBS ( $\mathrm{pH}$ 7.2). The antigenic sites were enhanced by incubation of sections in boiling citrate buffer (11 mM, pH 6.0) for $15 \mathrm{~min}$. Next, endogenous peroxidase activity was blocked by incubating the sections for 10 min in $3 \% \mathrm{H}_{2} \mathrm{O}_{2}$ in methanol, followed by blocking with $5 \% \mathrm{BSA}$ in PBS for $1 \mathrm{~h}$ at $37^{\circ} \mathrm{C}$ to reduce nonspecific binding of the first antibody. Sections were then incubated with Anti-CIRP (ab106230, Abcam; dilution 1:100 in PBS) antibody at $4^{\circ} \mathrm{C}$ overnight. After labelling with primary antibody, sections were washed with PBS-Tween $0.1 \%$ and incubated with horseradish peroxidase conjugated mouse anti-goat IgG (sc-2354, Santa Cruz) in room temperature. The immunoperoxidase colour reaction was developed with diaminobenzidene (DAB). The sections were lightly counterstained with haematoxylin, dehydrated in increasing concentrations of ethanol, cleared and covered with mounting medium and coverslips. To assess the specificity of the immunolabelling, positive controls consisted of mouse testis sections following manufacturer's protocol of Anti-CIRP antibody (ab106239), and as a negative control active primary antibody was replaced by the main antibody with BSA. All other steps and conditions remained the same.

\section{Data analysis of RT-PCR and Western blot}

Statistical analyses were performed using SPSS21 software (Statistical Analysis System Inc., Cary, NC, USA). The relative expression of CIRP mRNA and proteins in different tissues of adult yaks was analysed using one-way ANOVA procedure of SPSS 21.0 (SPSS Inc., Chicago, IL, USA). Data were expressed as means \pm standard errors. A probability of $p<0.05$ was considered to be the statistically significant. 


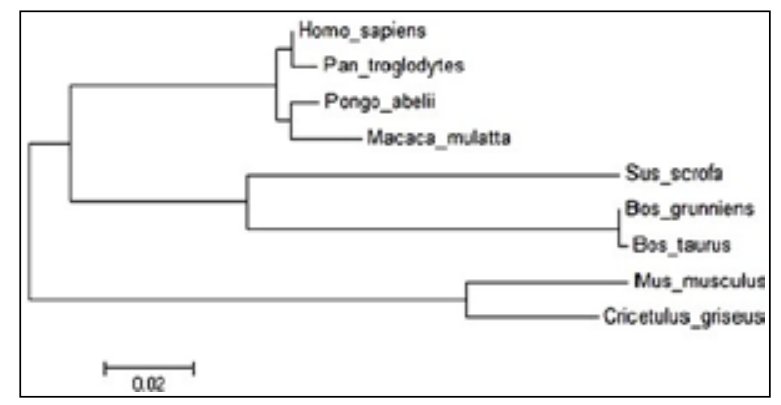

Figure 2. The phylogenetic tree of yak cold-inducible RNA binding protein (CIRP) with its closely related counterparts based on the CIRP cDNA sequences.

\section{RESULTS}

\section{Cloning and characterisation of CIRP open reading frame from domestic yak}

The obtained cDNA sequence of yak CIRP comprises 2197 bp containing an open reading frame (ORF) of 642 bp starting with an ATG (adenine, thymine, guanine) at position 77 and ending with a TGA (thymine, guanine, adenine) at position 718, encoded for 213 amino acids and their molecular weight was estimated as $23 \mathrm{kDa}$. The yak CIRP cDNA sequence has been deposited in the GenBank with accession no. KF682140.

The multiple alignment of cDNA sequences of yak CIRP with other species revealed significant homology of $99.8 \%$ with Bos taurus, $83.5 \%$ with Homo sapiens, $83.5 \%$ with Sus scrofa, $83.0 \%$ with Pongo abelii, $82.8 \%$ with Pan troglodytes, $82.4 \%$ with Macaca mulatta, $75.5 \%$ with Cricetulus griseus and $75.0 \%$ with Mus musculus. A phylogenetic tree (Fig. 2) was constructed in order to investigate the evolutionary relationship of yak CIRP with its closely related counterparts based on the CIRP cDNA sequences.

\section{CIRP mRNA expression in domestic yak}

Using real-time RT-PCR, we found that CIRP was widely expressed in different tissues (heart, liver, spleen, lung, kidney, brain, ovary, testis and skin) of the domestic yak only differing in quantity. The CIRP mRNA was highly expressed in the heart, kidney and skin of the domestic yak. Its mRNA expression in the heart was about 1.3 fold as much as in the kidney and skin. Its mRNA expression in the heart was about 2.5-2.8 fold as much as in the brain, testis and spleen. And its mRNA expression in the heart was about 6.7 fold as much as in the liver and also it was about 10 fold as much as in the lung and ovary (Fig. 3).

\section{CIRP protein expression in domestic yak}

Through Western blot on the total protein of the 9 tissues, we found that CIRP was widely expressed in them (heart, liver, spleen, lung, kidney, brain, ovary, testis and skin) only differing in quantity. CIRP protein was highly expressed in the heart, kidney, testis, skin and brain. CIRP protein expression in the heart was about 1.2-1.4 fold as much as in the kidney, testis, skin and brain. Its protein expression in the heart was about 2.0-2.5 fold as much as in the spleen, liver and ovary. And its protein expression in the heart was about 20 fold as much as in the lung (Fig. 4).

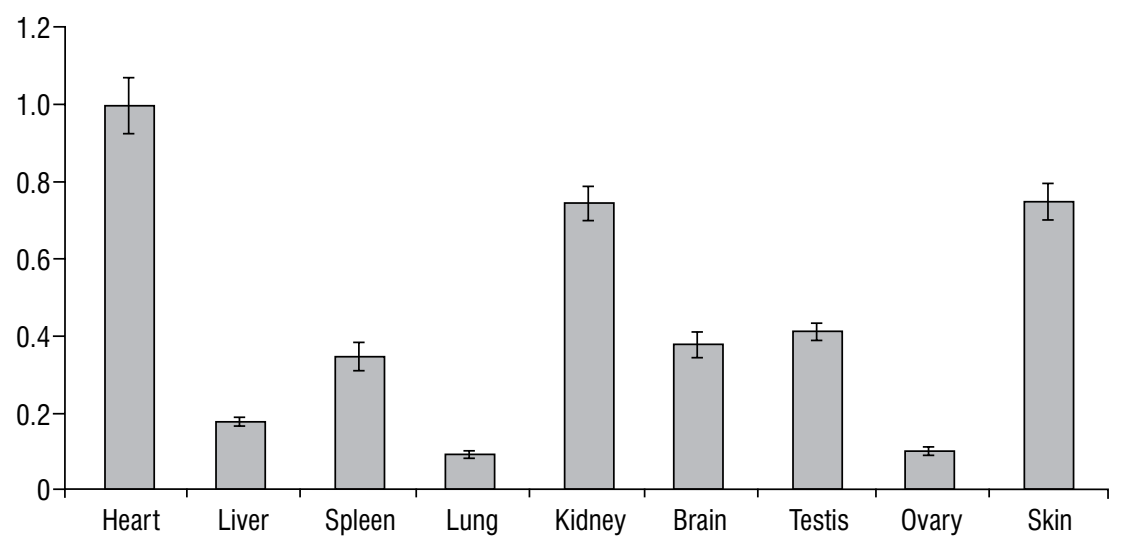

Figure 3. Expression of cold-inducible RNA binding protein (CIRP) mRNA in different tissues of the domestic yak (real-time polymerase chain reaction [real time RT-PCR] results). The figure is the ratio of different tissues CIRP gene copies to $\beta$-actin gene copies in $5 \mu \mathrm{g}$ total RNA of the domestic yak. Representative results from four independent experiments in triplicate on the same mRNA of different domestic yak are presented as means \pm standard errors. 


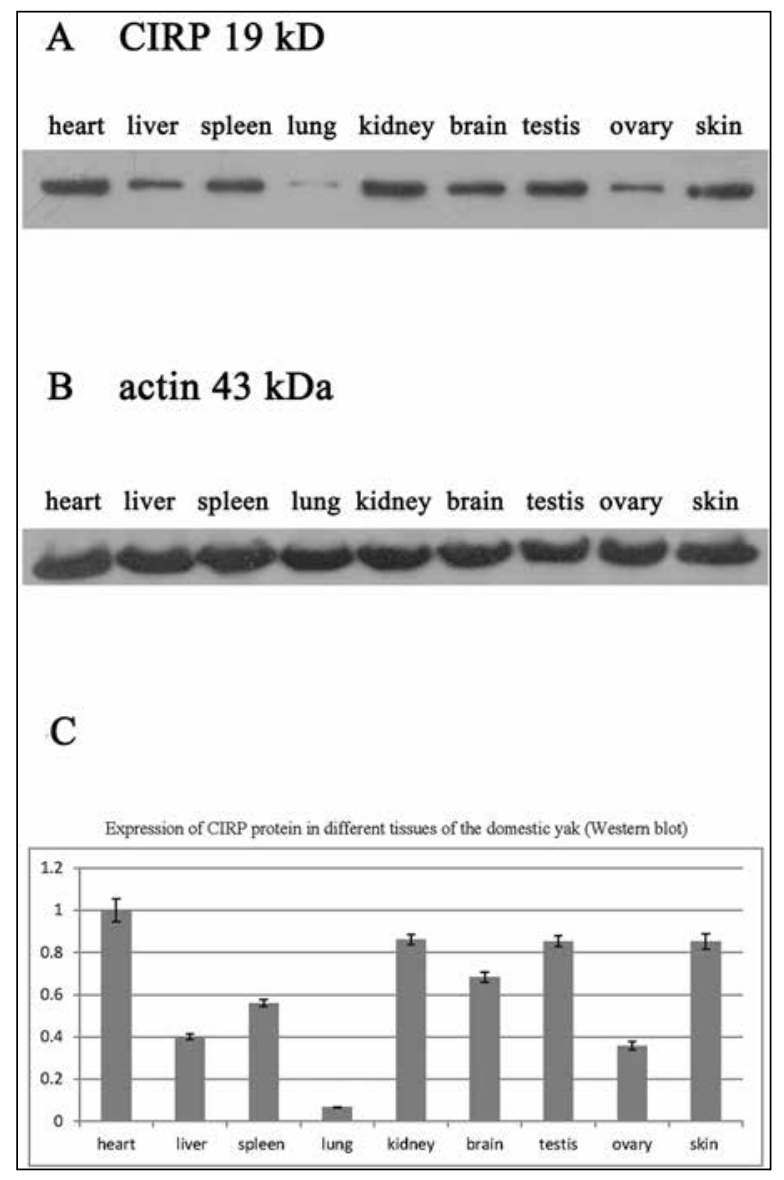

Figure 4. Expression of cold-inducible RNA binding protein (CIRP) in different tissues of the domestic yak. The actin protein was used as the standard to determine the quantity of the total protein. The over picture in panel $\mathbf{A}$ is the CIRP polyclonal antibody result and its molecular weight is about $19 \mathrm{kDa}$. The picture in panel $\mathbf{B}$ is the actin polyclonal antibody result and its molecular weight is about $43 \mathrm{kDa}$. C. The ratio of CIRP protein expressed quantity to actin protein expressed quantity calculated by the software ImageJ. Representative results from three independent experiments on the same protein of different domestic yak are presented as means \pm standard errors.

\section{Immunohistochemistry of CIRP}

We detected CIRP was constitutively expressed in heart, liver, spleen, lung, kidney, brain, ovary, testis and skin (Fig. 5). Positive reaction was more abundant in the cytoplasm of yak heart cells (Fig. 5A). Moreover, the cytoplasm of liver cells was widely labelled (Fig. 5B). In the spleen, positive cells were observed in red pulp and further study should be made to identify the cell type (Fig. 5C). And in the lung, CIRP was mainly detected in the cytoplasm of epithelial cells of trachea (Fig. 5D). Additionally, the expression in the cytoplasm of proximal and distal tubules was

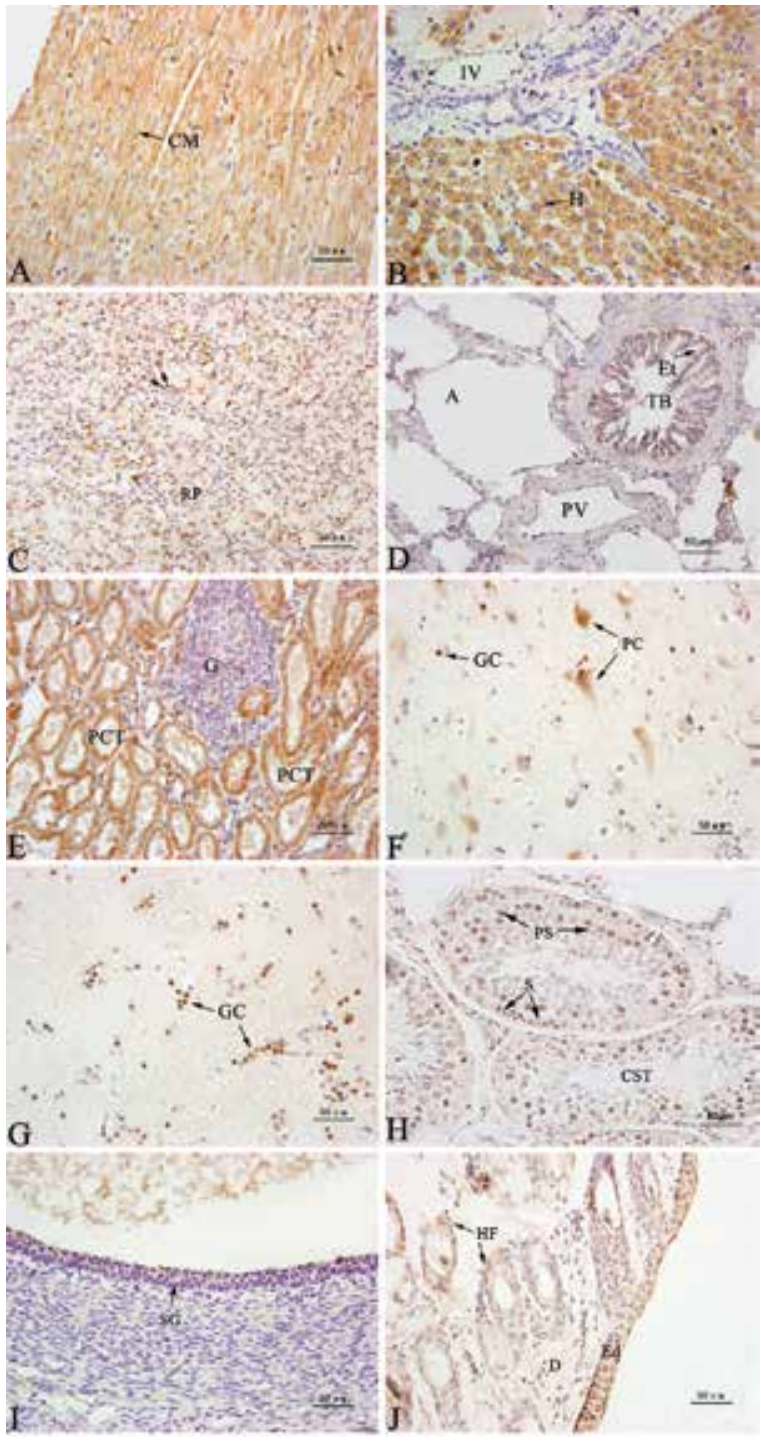

Figure 5. Immunohistochemical staining of cold-inducible RNA binding protein in yak heart (A), liver (B), spleen (C) (the arrows refers to the positive cells), lung $(\mathbf{D})$, kidney $(\mathbf{E})$, brain $(\mathbf{F}, \mathbf{G})$, testis $(\mathbf{H})$, ovary (I) and skin (J); $\mathrm{CM}$ - cardiac muscle; $\mathrm{H}$ - hepatocyte; IV — interlobular veins; RP — red pulp; TB — terminal bronchiole; Et — epithelium; A — alveolus; PV — pulmonary vein; G - glomeruli; PCT — proximal convoluted tubule; PC — pyramidal cell; $\mathrm{GC}$ - granular cell; CST — convoluted seminiferous tubules; PS primary spermatocyte; $S$ - spermatogonia; $S G$ - stratum granulosum; Ed - epidermis; D - dermis; HF - hair follicle; bar $=50 \mu \mathrm{m}$.

obvious except for renal corpuscles (Fig. 5E). And in yak brain, CIRP marked mainly the cytoplasm of pyramidal cells and the nuclei of granular cells (Fig. 5F, G). Especially, CIRP was detected in convoluted seminiferous tubules, and the nucleus of primary spermatocytes and spermatogonias was highly immunolabelled (Fig. 5H). In the yak ovary, CIRP was expressed in the cytoplasm of granular cells (Fig. 5I). 
And in the skin (skin of dorsum), the expression in the cytoplasm of the hair follicle, and also presented in the cytoplasm and nucleus of epidermal cells (Fig. 5J).

\section{DISCUSSION}

Cold-inducible RNA binding protein is the first cold shock protein identified in mammalian cells and its expression is suppressed by elevated temperatures [7]. In this study, we have isolated the yak CIRP coding sequence, and found that the encoded protein was $100 \%$ identical in amino acid sequence to that of cow [17], so that we summarise both structure of nucleic acids and amino acids are highly conservative and homologous.

Additionally, it is reported that CIRP is constitutively expressed in the testis, lung, heart, kidney, hippocampus and cerebral cortex of the adult rat [15]. In this study, when comparing the expression level of CIRP mRNA and protein from female and male yaks' tissues, no significant sex-differences were found. Additionally, we found CIRP mRNA and protein were differently expressed in heart, liver, spleen, lung, kidney, brain, ovary, testis and skin of adult yaks. Interestingly, the high expression level of CIRP was in the heart, kidney, brain, testis and skin. This phenomenon suggests that CIRP accumulates in stress granules upon exposure to a variety of stresses (e.g., hypoxia, cold, UV and osmotic pressure), and CIRP might regulate adaptation to plateau. Then previous investigations showed that the level of CIRP mRNA in mice testis was the highest than other various tissues [16], but our data showed CIRP level in yak hearts was the highest. Wellmann et al. [13] showed hypoxic transduction pathways shared common components with cold-stress transduction pathways, and expression of CIRP was stimulated under hypoxic conditions by a hypoxia inducible transcription factor (HIF)-1 independent mechanism. Moreover, we propose the highest levels of CIRP in yak heart might be able to make yaks respond and adapt quickly to hypoxia through a myriad of mechanisms. Interestingly, Sugimoto and Jiang [11] reported the CIRP expression level in tree frog heart and kidney accounted for only from $20 \%$ to $30 \%$ of the brain. This is different from our data that showed CIRP expression in yak brain was lower than in heart, skin and kidney. However, the previous study showed the level of CIRP mRNA in the brain was diurnally regulated [8], and it increased during the daytime, reaching the highest level at 18:00, and then decreased to the lowest at 03:00.
So we conclude that the lower CIRP expression in yak brain might be due to the brain sample taking at 04:00. Additionally, The outermost layer of the body, the skin is easily exposed to cold and sunlight. CIRP protein levels increased in a dose-dependent manner in response to UV, and cells that expressed reduced CIRP levels were more sensitive to UV radiation [5]. So we suspect the high levels of CIRP in yak skin have cytoprotective effects against UV. Recently, the induction of CIRP in lung was observed, when mice are kept under experimental hypoxic conditions [5]. We also observed the expression in yak's lung under native hypoxic conditions. Whether this is mediated via the binding site for the HIF-1 present in the CIRP gene or other pathways is under investigation. At present, studies about the CIRP expression in mammalian have barely concentrated on other tissues such as liver, spleen, kidney, lung and ovary. In this study we firstly identify the differences in CIRP expression of various tissues, and the regulatory mechanisms of CIRP in various tissues is still unclear.

Our immunohistochemical data demonstrated CIRP was constitutively expressed in the cells of yak's different tissues, corresponding with those found in murine and human. Particularly, we found a high level of CIRP protein was detected immunohistochemically in the nucleus of spermatogonia and primary spermatocytes of testis, corresponding with those found in mouse [6]. We extrapolate this protein might play important roles in regulating male germ cells on the differentiation stage. In addition, we detected the CIRP protein was expressed in cardiac muscle cells, epithelial cells of trachea and epidermal cells of skin. It is easily inferred that the environmental stress in Qinghai-Tibet plateau, including cold temperature, low oxygen content and strong ultraviolet ray, could induce the expression of CIRP. Brain cells, especially neurons such as pyramidal cells and granular cells, are vulnerable to various injuries for instance hypoxia, trauma, and infection [2]. Immunohistochemical analysis shows that CIRP is expressed in the nucleus of neurons. It is tempting to hypothesize that the strong positive stainings for CIRP in yak brain have cytoprotective effects against injuries and may play a role in biological rhythms. Moreover, CIRP was positively presented in ovarian granular cells, the kidney tubules, the red pulp, the liver cells, although the particular physiological function of CIRP was still not clear. Production of CIRP-gene knockout mice is in progress, and expected to clarify various functions of CIRP. 
Remarkably, we detected CIRP was expressed both in the nucleus of neuronal cells, spermatogonia and epidermal cells, and in the cytoplasm of residual tissues. While cold-shock does not modify the localisation of CIRP, upon exposure to a variety of stresses (e.g., osmotic pressure). CIRP translocates from the nucleolus to the cytoplasm. CIRP proteins could thus play different roles both in the nucleus and the cytoplasm. At the nuclear level, CIRP might play an important role in cold-induced suppression of cell growth [7]. In the cytoplasm, the association of $x \mathrm{CIRP} 2$ with the RNA-binding protein ElrA might regulate the length of the poly (A) tail of specific mRNAs [1].

\section{CONCLUSIONS}

In conclusion, this is the first report on the wide expression of CIRP mRNA and protein in the different tissues of the native plateau animal. In this study we obtained CIRP ORF of domestic yak and found that both CIRP mRNA and protein of the domestic yak are tissue specific expression. Especially the specific expression of CIRP protein may give us the useful information to understand and further study the important role of CIRP protein in the adaptation to the high altitude of the domestic yak on long-term evolution. It should be moreover determined whether the season of the year could influence the CIRP expression.

\section{Acknowledgements}

The authors thank the support from the National Natural Science Foundation of China (grant no. 31360594).

\section{REFERENCES}

1. Aoki K, Matsumoto K, Tsujimoto M (2003) Xenopus cold-inducible RNA-binding protein 2 interacts with ElrA, the Xenopus homolog of HuR, and inhibits deadenylation of specific mRNAs. J Biol Chem, 278: 48491-48497.

2. de-Zwart LL, Meerman JH, Commandeur JN, Vermeulen NP (1999) Biomarkers of free radical damage: applications in experimental animals and in humans. Free Radical Bio Med, 26: 202-226.

3. Fleige S, Pfaffl MW (2006) RNA integrity and the effect on the real-time qRT-PCR performance. Mol Aspects Med, 27: 126-139.

4. Gang C, Tiancai L, Hongan L, Luojun X (1991) The anatomic characteristics of the yak heart and lung. Chinese Yak, 2: 007.
5. Lleonart M (2010) A new generation of proto-oncogenes: cold-inducible RNA binding proteins. BBA-Rev Cancer, 1805: 43-52.

6. Nishiyama H, Danno S, Kaneko $Y$, Itoh $K$, Yokoi H, Fukumoto M, Okuno H, Millan JL, Matsuda T, Yoshida O, Fujita J (1998) Decreased expression of cold-inducible RNA-binding protein (CIRP) in male germ cells at elevated temperature. Am J Pathol, 152: 289-296.

7. Nishiyama H, Itoh K, Kaneko Y, Kishishita M, Yoshida O, Fujita J (1997) A glycine-rich RNA-binding protein mediating cold-inducible suppression of mammalian cell growth. J Cell Bio, 137: 899-908.

8. Nishiyama $H$, Xue $J H$, Sato $T$, Fukuyama $H$, Mizuno $N$, Houtani T, Sugimoto T, Fujita J (1998) Diurnal change of the cold-inducible RNA-binding protein (Cirp) expression in mouse brain. Biochem Biophys Res Commun, 245: 534-538.

9. Ruscher K, Isaev N, Trendelenburg G, Weih M, lurato L, Meisel A, Dirnagl U (1998) Induction of hypoxia inducible factor 1 by oxygen glucose deprivation is attenuated by hypoxic preconditioning in rat cultured neurons. Neurosci Lett, 254: 117-120.

10. Schmittgen TD, Zakrajsek BA, Mills AG, Gorn V, Singer MJ, Reed MW (2000) Quantitative reverse transcription-polymerase chain reaction to study mRNA decay: comparison of endpoint and real-time methods. Anal Biochem, 285: 194-204.

11. Sugimoto $K$, Jiang $H(2008)$ Cold stress and light signals induce the expression of cold-inducible RNA binding protein ( cirp) in the brain and eye of the Japanese treefrog (Hyla japonica). Comp Biochem Phys A, 151: 628-636 .

12. Wang DP, Li HG, Li YJ, Guo SC, Yang J, Qi DL, Jin C, Zhao XQ (2006) Hypoxia-inducible factor 1 alpha cDNA cloning and its mRNA and protein tissue specific expression in domestic yak (Bos grunniens) from Qinghai-Tibetan plateau. Biochem Biophys Res Commun, 348: 310-319.

13. Wellmann S, Buhrer C, Moderegger E, Zelmer A, Kirschner R, Koehne P, Fujita J, Seeger K (2004) Oxygen-regulated expression of the RNA-binding proteins RBM3 and CIRP by a HIF-1-independent mechanism. J Cell Sci, 117: 1785-1794.

14. Wiener G, Han JL, Long RJ (2003) The Yak 2nd edn. Regional Office for Asia and the Pacific Food and Agriculture Organization of the United Nations, Bangkok.

15. Xue JH, Nonoguchi K, Fukumoto $M$, Sato T, Nishiyama $H$, Higashitsuji H, Itoh K, Fujita J (1999) Effects of ischemia and $\mathrm{H} 2 \mathrm{O} 2$ on the cold stress protein CIRP expression in rat neuronal cells. Free radical Bio Med, 27: 1238-1244.

16. Zhou KW, Zheng XM, Yang ZW, Zhang L, Chen HD (2009) Overexpression of CIRP may reduce testicular damage induced by cryptorchidism. Clin Invest Med, 32: E103-E111.

17. Zimin AV, Delcher AL, Florea L, Kelley DR, Schatz MC, Puiu D, Hanrahan F, Pertea G, Van Tassell CP, Sonstegard TS, Marcais G, Roberts M, Subramanian P, Yorke JA, Salzberg SL (2009) A whole-genome assembly of the domestic cow, Bos taurus. Genome Biol, 10: R42. 